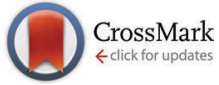

Cite this: Chem. Commun., 2015, 51, 6168

Received 12th February 2015, Accepted 3rd March 2015

DOI: $10.1039 / c 5 c c 01315 a$

www.rsc.org/chemcomm

\section{Adsorption and desorption characteristics of alcohol vapors on a nanoporous ZIF-8 film investigated using silicon microcantilevers $\uparrow$}

\author{
Changyong Yim, ${ }^{a}$ Moonchan Lee, ${ }^{a}$ Wuseok Kim, ${ }^{a}$ Sanghee Lee, ${ }^{a}$ Gook-Hee Kim, ${ }^{b}$ \\ Kyong Tae Kim ${ }^{b}$ and Sangmin Jeon*a
}

\begin{abstract}
Zinc oxide nanorods were synthesized directly on a silicon microcantilever and converted into a nanoporous ZIF-8 film via a solvothermal reaction. The simultaneous measurements of the resonance frequency and deflection of the cantilever revealed that the adsorption of alcohol vapors induced a structural change in the ZIF-8 framework.
\end{abstract}

Zeolitic imidazolate frameworks (ZIFs), a subset of metal-organic frameworks (MOFs) or porous coordinated polymers, have attracted much attention recently due to their superior chemical and thermal stabilities compared to conventional MOFs. The porosity and chemical functionalities of ZIFs can be easily tailored by tuning the central metal ions and the organic linkers, ${ }^{1}$ thereby creating materials that are useful in traditional applications such as gas storage, gas separation, and heterogeneous catalysis, ${ }^{2-5}$ as well as in chemical vapor sensing applications. ${ }^{6-9}$ The integration of ZIFs into gas sensing platforms, however, is not straightforward because conventional gas sensors measure changes in electrical properties, whereas most ZIFs are not conductive. ${ }^{10}$

This problem may be overcome by using ZIFs in combination with mechanical transducers, such as microcantilevers, as sensing platforms. Microcantilevers have two operation modes, a static mode and a dynamic mode, that detect changes in the deflection and resonance frequency, respectively, induced by the adsorption of gas molecules onto the cantilever surface. ${ }^{11}$ The sensitivity of a microcantilever is determined by its dimensions, whereas the selectivity of a microcantilever sensor is achieved by applying a particular coating material on the sensor surface., ${ }^{72}$ Among the various materials available as sensing materials, polymers are preferred because their chemical composition may be tuned and they are easy to apply to the cantilever surface. ${ }^{13}$ The drawback to using polymers as sensing materials is that their response to the

\footnotetext{
${ }^{a}$ Department of Chemical Engineering, Pohang University of Science and

Technology (POSTECH), Pohang, Gyeongbuk, Republic of Korea.

E-mail: jeons@postech.ac.kr

${ }^{b}$ Clean Coal Chemicals Research Project, Research Institute of Industrial Science and Technology (RIST), Pohang, Gyeongbuk, Republic of Korea

$\dagger$ Electronic supplementary information (ESI) available: A schematic illustration of the instrument set-up. See DOI: 10.1039/c5cc01315a
}

adsorption and desorption of gas molecules is slow, which degrades the sensor performance. By contrast, ZIFs respond rapidly to the binding and release of gas molecules due to their high porosity. ${ }^{7}$ In addition, the large surface area and tunable chemical composition of ZIF films suggest that ZIFs could be excellent sensing materials that, when coupled with microcantilever sensors, would yield high sensitivity and selectivity and rapid response times.

Several methods have been developed for coating MOFs on sensor surfaces. In order to measure simultaneously the responses from the dynamic mode and static mode of a microcantilever sensor, only one side of the cantilever surface should be coated, which limits the application of conventional dip coating techniques. Hwang et al. used photolithography techniques to pattern molds with a square cross-section that permitted drop-casting of a ZIF layer onto a bridge microresonator. ${ }^{14}$ Allendorf and Hesketh formed a self-assembled monolayer on gold-coated microcantilevers and synthesized a HKUST-1 framework on only the monolayercoated surfaces. ${ }^{15}$

In this study, we developed a simple method for synthesizing ZIF-8 films on one side of silicon microcantilevers and examined the response of the ZIF cantilever to the adsorption of alcohol vapor. A thin layer of zinc oxide (ZnO) was sputtered onto a silicon wafer and a silicon microcantilever. ZnO nanorods were synthesized directly on the substrates via solvothermal reactions, and these layers were converted into ZIF-8 films. Unlike previous methods, which used vacuum-deposited $\mathrm{ZnO}$ films as the metal ion sources and required atomically smooth sensing surfaces to produce a highly crystalline framework, ${ }^{16}$ the ZIF-8 films obtained from the $\mathrm{ZnO}$ nanorods exhibited a high crystallinity, even on the rough silicon microcantilever surfaces. Exposure of the ZIF-8 film-grown microcantilevers (ZIF cantilevers) to methanol, ethanol, or 1-propanol induced changes in the resonance frequency and deflection of the cantilever. Simultaneous measurements of the resonance frequency and cantilever deflection revealed that the ZIF-8 layer undergoes structural changes during alcohol vapor adsorption, and that these changes depend on the alcohol vapor concentration. 


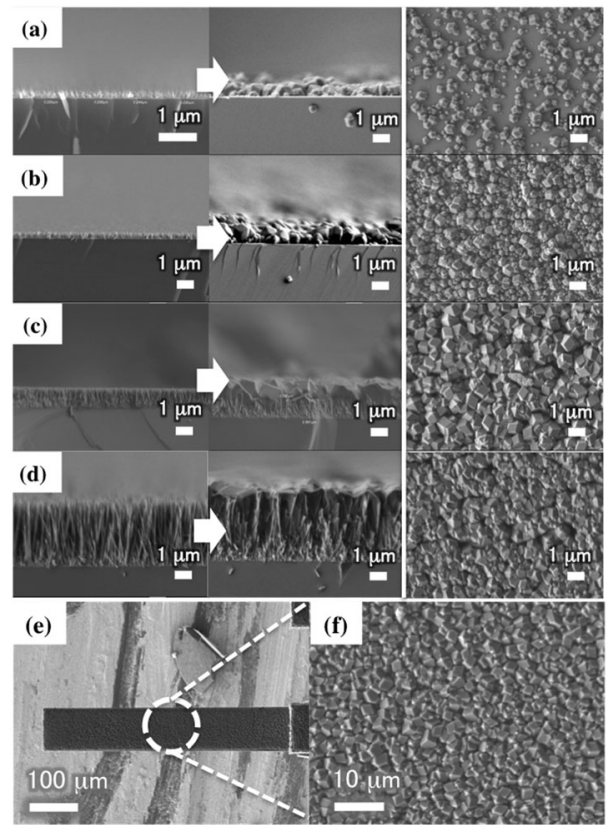

Fig. 1 Side-view SEM images of the $\mathrm{ZnO}$ nanorods grown with different lengths ((a) $250 \mathrm{~nm}$, (b) $360 \mathrm{~nm}$, (c) $1.2 \mu \mathrm{m}$, (d) $3.5 \mu \mathrm{m}$ ) on a silicon wafer, and side-view and top-view SEM images of the corresponding ZIF-8 films after $1 \mathrm{~h}$ solvothermal reactions. (e) Top-view SEM image of a ZIF cantilever, and (f) magnified image of the ZIF cantilever surface.

The first column in Fig. 1(a)-(d) shows side-view scanning electron microscopy (SEM) images of $\mathrm{ZnO}$ nanorods with different lengths between $250 \mathrm{~nm}$ and $3.5 \mu \mathrm{m}$ grown on a silicon wafer. After conversion of the $\mathrm{ZnO}$ nanorods to ZIF-8 films via a solvothermal reaction, the film thickness increased substantially because the molar volume of ZIF-8 was 17 times larger than that of $\mathrm{ZnO}$ (see the second column in Fig. 1(a)-(d) for side-view images of the ZIF-8 films). ${ }^{17}$ The top-view SEM images of the ZIF-8 films in the third column of Fig. 1(a)-(d) show that the ZIF-8 films consisted of granules with diameters of a few hundred nanometers. The granule size increased with the initial $\mathrm{ZnO}$ nanorod length because the larger $\mathrm{ZnO}$ nanorods provided more zinc ions for the solvothermal reaction, indicating that the concentration of zinc ions limited the growth of the ZIF-8 films under our experimental conditions. The $250 \mathrm{~nm} \mathrm{ZnO}$ nanorods (Fig. 1(a)) produced a ZIF-8 film that partially covered the surface, and the $360 \mathrm{~nm} \mathrm{ZnO}$ nanorods (Fig. 1(b)) produced a ZIF-8 film that was apparently weakly bound to the surface. However, the $1.2 \mu \mathrm{m} \mathrm{ZnO}$ nanorods showed the uniform ZIF-8 film (Fig. 1(c)); these nanorods were used for the synthesis of ZIF-8 films in all subsequent XRD characterization and gas sensing applications. Fig. 1(e) and (f) show SEM images of the ZIF cantilever at different magnifications. The conversion of the $\mathrm{ZnO}$ nanorod layer to the ZIF-8 layer increased the film thickness from $1.2 \mu \mathrm{m}$ to $2.2 \mu \mathrm{m}$. Note that the ZIF-8 film was synthesized only one side of the silicon cantilever, enabling the observation of cantilever deflections during gas adsorption. $\neq \S$

Fig. 2 shows the XRD patterns of a $30 \mathrm{~nm}$ thick $\mathrm{ZnO}$ seed layer (black), ZnO nanorods after the hydrothermal reactions (blue), and the resulting ZIF-8 film after the solvothermal reactions (red).

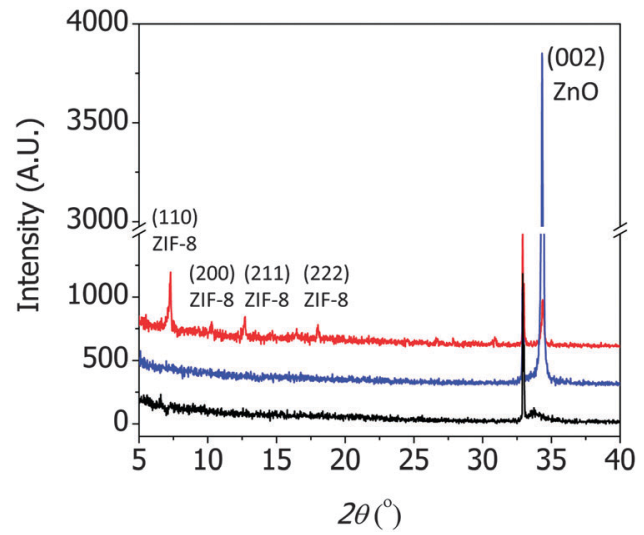

Fig. 2 X-ray diffraction (XRD) patterns of the $\mathrm{ZnO}$ seed layer (black), ZnO nanorods (blue), and ZIF- 8 film synthesized from the $\mathrm{ZnO}$ nanorods (red).

The ZnO seed layer showed a strong peak at $32.9^{\circ}$ corresponding to the (112) plane of the Ti adhesion layer and a weak peak at $33.7^{\circ}$ corresponding to the (002) plane of the $\mathrm{ZnO}$ film. ${ }^{18,19}$ The ZnO nanorods showed a strong (002) peak, suggesting that they grew perpendicularly to the substrate. The ZIF-8 film showed several peaks at $7.3^{\circ}, 10.4^{\circ}, 12.7^{\circ}$, and $18.02^{\circ}$, corresponding to the (100), (200), (211), and (222) peaks of ZIF-8, according to the CCDC number $602542 .{ }^{1}$ Note that the presence of the (002) ZnO peak in the ZIF-8 film indicated that the $\mathrm{ZnO}$ nanorods were not completely converted to ZIF. The extent of conversion was intentionally controlled to achieve strong adhesion between the ZIF-8 film and the silicon substrate. $\S$

Fig. 3 shows the resonance frequency of the ZIF cantilever during the adsorption and desorption of saturated methanol, ethanol, or 1-propanol vapor. The adsorption of alcohol vapor onto the surface of the cantilever instantly decreased the resonance frequencies (indicating an increase in the mass). The resonance frequency of a cantilever is directly related to the adsorbed mass on the cantilever according to

$$
f=\frac{1}{2 \pi} \sqrt{\frac{k}{m^{*}}}
$$

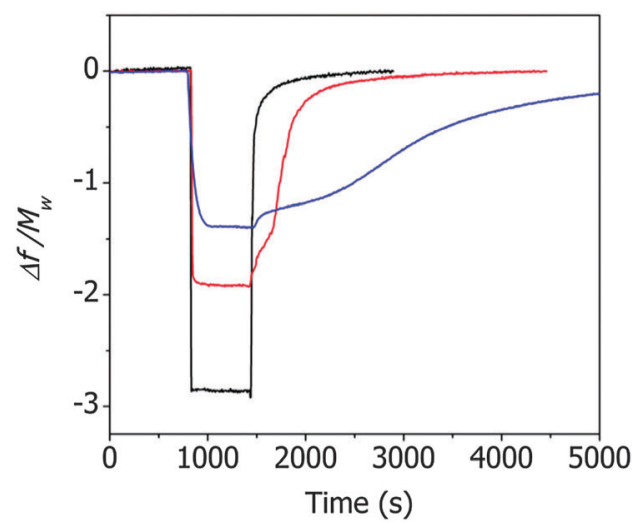

Fig. 3 Resonance frequency of the ZIF cantilever normalized by the molecular weight of the alcohol during the adsorption and desorption of saturated methanol (black), ethanol (red), or 1-propanol (blue) vapor. 
where $f, m^{*}$, and $k$ represent the resonance frequency, the effective mass, and the spring constant of the cantilever, respectively. In the present experiments, the actual mass of the adsorbed molecules was not calculated from the frequency change due to the uncertainty in the modulus of the ZIF-8 layer; however, it can be safely assumed that the frequency change will be correlated qualitatively with the mass change. The change in resonance frequency normalized by the molecular weight of the alcohol provided a measure of the number of adsorbed alcohol molecules. $\uparrow$

The magnitudes of the normalized frequency changes upon vapor adsorption followed the order of methanol $>$ ethanol $>$ 1-propanol and were inversely proportional to the molecular weight of the alcohol molecules. The pore size of ZIF-8 was $1.2 \mathrm{~nm},{ }^{20}$ and the aperture sizes of the ZIF- 8 varied from $0.35 \mathrm{~nm}$ to $0.58 \mathrm{~nm}$, depending on the gas adsorption-induced structural changes of the framework. ${ }^{21}$ The molecular sizes of methanol, ethanol, and 1-propanol are $0.36 \mathrm{~nm}, 0.45 \mathrm{~nm}$, and $0.47 \mathrm{~nm}$, respectively, ${ }^{22}$ and the largest change in the normalized resonance frequency was observed for methanol, which had the smallest molecular size. The desorption of methanol molecules from the ZIF-8 film induced a rapid change in the frequency, indicating that the interactions between methanol and the ZIF-8 film were not strong. By contrast, the desorption of ethanol or 1-propanol molecules from the ZIF-8 film induced sluggish changes in the frequency, and a slower response was observed from the desorption of 1-propanol than of ethanol. These results indicated that the alcohol vapor molecules adsorbed or desorbed mainly at the hydrophobic imidazole linkers. ${ }^{23}$

The changes in the deflection of the ZIF cantilever were measured simultaneously with the changes in the resonance frequency (see Fig. 3), as shown in Fig. 4. The largest deflection was observed during the adsorption of methanol, indicating that the many more methanol molecules were adsorbed to the cantilever compared to ethanol and 1-propanol; however, the cantilever deflections due to the adsorption of 1-propanol were larger than the deflections induced by ethanol adsorption, even though the number of adsorbed 1-propanol molecules was smaller. This result suggests that the deflection of the cantilever is affected not only by the number of alcohol molecules but also by the interactions between the adsorbed alcohol molecules and the

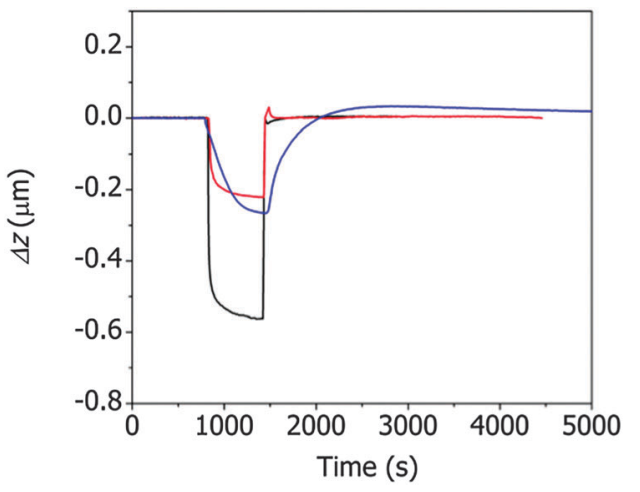

Fig. 4 Deflection of the ZIF cantilever during the adsorption and desorption of saturated methanol (black), ethanol (red), or 1-propanol (blue) vapor.
ZIF surface. The strong adsorption of 1-propanol resulted in slower recovery of the deflection during its desorption compared to ethanol or methanol.

It is interesting to note that, during desorption of the alcohol molecules, the changes in deflection (Fig. 4) differed significantly from the changes in resonance frequency (Fig. 3). Specifically, the resonance frequency changes during desorption lasted longer than the deflection changes during desorption. This unusual result can be attributed to the presence of two different types of alcohol molecules inside ZIF-8 pores: molecules adsorbed onto the surface of ZIF-8 and free molecules not bound to the surface. The adsorbed molecules affected both the resonance frequency and the cantilever deflection, whereas the unbound molecules affected only the resonance frequency of the cantilever. In this situation, the longer time for the recovery of the resonance frequency compared to the deflection indicates that the desorption process was diffusion-limited. The separation of the adsorbed molecules from the ZIF surface was faster than the rinsing of the separated molecules out of the pores.

Fig. 5(a) shows the variations in the normalized resonance frequency of the ZIF cantilever as a function of the vapor concentrations of methanol, ethanol, and 1-propanol. For each alcohol, the frequency changes increased with the concentration and displayed S-shaped isotherms at room temperature. The alcohol vapor concentration at which the abrupt change occurred was defined as the critical concentration. The critical concentrations are associated with the gate opening-induced structural changes in the framework and determined for methanol, ethanol, and 1-propanol to be $10 \%, 5 \%$, and $3 \%$, respectively, which agreed well with the results reported by Remi et $a l^{24,25}$ The critical concentration decreased as the hydrophobicity of the adsorbed alcohol molecules increased, further indicating that the ZIF-8 films were hydrophobic.

Fig. 5(b) shows the deflections measured simultaneously with the resonance frequencies of the ZIF cantilever upon the adsorption of various concentrations of methanol, ethanol, and 1-propanol. It is interesting to note that the cantilever bent upwards (i.e., toward the ZIF-8 film) at low concentrations, but bent downwards (i.e., away from the ZIF-8 film) at high concentrations. The alcohol vapor concentrations at which the cantilevers began to bend upward coincided with the critical concentrations determined from the resonance frequency measurements, indicating that tensile stresses formed during the
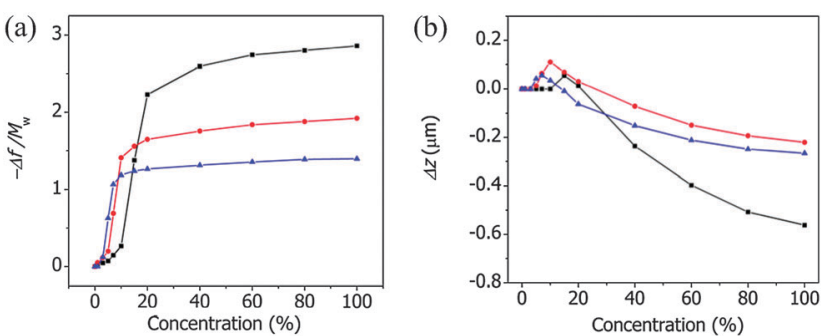

Fig. 5 The normalized resonance frequency (a) and the deflection (b) of the ZIF cantilever due to the adsorption of various concentrations of $\mathrm{MeOH}$ (black), EtOH (red), or 1-propanol (blue). 
gate opening of the ZIF-8 film. The further adsorption of alcohol molecules onto the ZIF-8 film caused the framework to expand (i.e., bend downward), and the balance between the two effects produced maximal concentration-dependent deflection changes.

We developed a facile method for synthesizing nanoporous ZIF-8 layers directly on silicon microcantilevers by using a $\mathrm{ZnO}$ nanorod layer as a metal ion source. The volume expansion due to the conversion of the $\mathrm{ZnO}$ nanorods to a ZIF-8 film was accommodated by the open spaces between the $\mathrm{ZnO}$ nanorods, which prevented excessive bending of the cantilever during the growth of the ZIF-8 layer. The ZIF cantilever was exposed to methanol, ethanol, and 1-propanol vapor. The changes in the resonance frequency and deflection of the cantilever were measured simultaneously during the adsorption and desorption of these alcohol vapors onto the ZIF-8 layer. The critical alcohol vapor concentration needed to induce structural changes in the framework was found to decrease in the order of 1-propanol $>$ ethanol $>$ methanol. This trend was attributed to differences in the concentration of alcohol required to induce gate opening of the ZIF-8 framework. The molecular weight-dependent adsorption and desorption kinetics of the alcohol vapors on the ZIF-8 may be used to separate a target alcohol from an alcohol vapor mixture.

This work was supported by Research Institute of Industrial Science and Technology (RIST), Pohang, Korea.

\section{Notes and references}

\$ Materials. Zinc nitrate hexahydrate $\left(\mathrm{Zn}\left(\mathrm{NO}_{3}\right)_{2} \cdot 6 \mathrm{H}_{2} \mathrm{O}\right)$, ammonium hydroxide solution, 2-methylimidazole (2-MIM), methanol, ethanol, and 1-propanol were purchased from Sigma-Aldrich and were used without further purification. Rectangular silicon microcantilevers were obtained from Micromotive (Mainz, Germany). The length, width, and thickness of each cantilever beam were $450 \mu \mathrm{m}, 90 \mu \mathrm{m}$, and $5 \mu \mathrm{m}$, respectively.

$\S$ Synthesis of the $\mathrm{ZnO}$ nanorods and their conversion into ZIF-8 films on silicon wafers and microcantilevers. Ti adhesion layers $(3 \mathrm{~nm})$ and ZnO seed layers $(30 \mathrm{~nm})$ were sputtered sequentially onto one side of the silicon wafers and silicon microcantilevers. ZnO nanorods were hydrothermally synthesized on the ZnO-deposited substrates over various periods of time in a $10 \mathrm{mM} \mathrm{Zn}\left(\mathrm{NO}_{3}\right)_{2}$ solution at a $\mathrm{pH}$ of 10.3 and $90{ }^{\circ} \mathrm{C}$. The substrates were then rinsed with deionized water and ethanol repeatedly and dried under a gentle nitrogen flow. The $\mathrm{ZnO}$ nanorod-coated substrates were UV-irradiated to produce hydroxyl groups on their surfaces. The conversion of $\mathrm{ZnO}$ to $\mathrm{ZIF}-8$ was governed by the competition between the dissolution and coordination of $\mathrm{Zn}^{2+} \cdot{ }^{16}$ To avoid the rapid dissolution of $\mathrm{ZnO}$ in the aqueous solution, the conversion was conducted by immersing the $\mathrm{ZnO}$ nanorod-grown substrates in a $1 \mathrm{M}$ 2-MIM methanol solution at $160{ }^{\circ} \mathrm{C}$ for $1 \mathrm{~h}$. The resulting ZIF-8 film-coated substrates were rinsed with deionized water and methanol and dried under a nitrogen flow. The $\mathrm{ZnO}$ nanorods and ZIF-8 films grown on silicon wafers were characterized by collecting side-view scanning electron microscopy (SEM) images and X-ray diffraction (XRD) patterns. The ZIF cantilever was used to monitor changes in the resonance frequency and deflection during the adsorption of alcohol vapors.

I Instrument set-up. Fig. S1 (ESI $\dagger$ ) shows a schematic illustration of the instrument set-up used in this study. The ZIF cantilever was mounted in a flow cell featuring an embedded piezoelectric actuator. A laser beam reflected from the silicon side of the cantilever was tracked using a position-sensitive detector, and changes in the deflection were monitored. The resonance frequency of the cantilever was determined using a home-built LabView program based on a fast Fourier transform (FFT) algorithm. ${ }^{26}$ Dry nitrogen was passed through a gas bubbler containing methanol, ethanol, or 1-propanol to generate vapor. The wet nitrogen flow containing the alcohol vapor was combined with a dry nitrogen stream, and the vapor mixture flow was injected into the flow cell. The gas flow rates of the dry and wet nitrogen were controlled using mass flow controllers (Brooks Instruments, Hatfield, PA), and the total flow rate was fixed at $100 \mathrm{~mL} \mathrm{~min}{ }^{-1}$. All experiments were conducted at room temperature.

1 K. S. Park, Z. Ni, A. P. Côté, J. Y. Choi, R. Huang, F. J. Uribe-Romo, H. K. Chae, M. O'Keeffe and O. M. Yaghi, Proc. Natl. Acad. Sci. U. S. A., 2006, 103, 10186.

2 P. Mishra, S. Edubilli, B. Mandal and S. Gumma, Microporous Mesoporous Mater., 2013, 169, 75.

3 J.-R. Li, R. J. Kuppler and H.-C. Zhou, Chem. Soc. Rev., 2009, 38, 1477.

4 H. Yang, X.-W. He, F. Wang, Y. Kang and J. Zhang, J. Mater. Chem., 2012, 22, 21849.

5 K. Zhang, R. P. Lively, M. E. Dose, A. J. Brown, C. Zhang, J. Chung, S. Nair, W. J. Koros and R. R. Chance, Chem. Commun., 2013, 49, 3245.

6 F. M. Hinterholzinger, A. Ranft, J. M. Feckl, B. Rühle, T. Bein and B. V. Lotsch, J. Mater. Chem., 2012, 22, 10356.

7 Y. Hwang, H. Sohn, A. Phan, O. M. Yaghi and R. N. Candler, Nano Lett., 2013, 13, 5271.

8 G. Lu, O. K. Farha, W. Zhang, F. Huo and J. T. Hupp, Adv. Mater., 2012, 24, 3970.

9 G. Lu and J. T. Hupp, J. Am. Chem. Soc., 2010, 132, 7832.

10 S. Eslava, L. Zhang, S. Esconjauregui, J. Yang, K. Vanstreels, M. R. Baklanov and E. Saiz, Chem. Mater., 2013, 25, 27.

11 R. Raiteri, M. Grattarola, H. J. Butt and P. Skladal, Sens. Actuators, B, 2001, 79, 115.

12 Y. Jin, D. Lee, S. Lee, W. Moon and S. Jeon, Anal. Chem., 2011, 83, 7194.

13 A. Loui, T. V. Ratto, T. S. Wilson, S. K. McCall, E. V. Mukerjee, A. H. Love and B. R. Hart, Analyst, 2008, 133, 608.

14 Y. Hwang, A. Phan, K. Galatsis, O. M. Yaghi and R. N. Candler, J. Micromech. Microeng., 2013, 23, 125027.

15 M. D. Allendorf, R. J. T. Houk, L. Andruszkiewicz, A. A. Talin, J. Pikarsky, A. Choudhury, K. A. Gall and P. J. Hesketh, J. Am. Chem. Soc., 2008, 130, 14404.

16 K. Khaletskaya, S. Turner, M. Tu, S. Wannapaiboon, A. Schneemann, R. Meyer, A. Ludwig, G. Van Tendeloo and R. A. Fischer, Adv. Funct. Mater., 2014, 24, 4804.

17 I. Stassen, N. Campagnol, J. Fransaer, P. Vereecken, D. De Vos and R. Ameloot, CrystEngComm, 2013, 15, 9308.

18 J. D. Hanawalt, H. W. Rinn and L. K. Frevel, Ind. Eng. Chem., Anal. Ed., 1938, 10, 457.

19 J. Joo, D. Lee, M. Yoo and S. Jeon, Sens. Actuators, B, 2009, 138, 485.

20 A. Demessence, C. Boissière, D. Grosso, P. Horcajada, C. Serre, G. Férey, G. J. A. A. Soler-Illia and C. Sanchez, J. Mater. Chem., 2010, 20, 7676.

21 K. Zhang, R. P. Lively, C. Zhang, R. R. Chance, W. J. Koros, D. S. Sholl and S. Nair, J. Phys. Chem. Lett., 2013, 4, 3618.

22 T. Borjigin, F. Sun, J. Zhang, K. Cai, H. Ren and G. Zhu, Chem. Commun., 2012, 48, 7613.

23 K. Zhang, L. Zhang and J. Jiang, J. Phys. Chem. C, 2013, 117, 25628.

24 J. Cousin Saint Remi, T. Rémy, V. Van Hunskerken, S. van de Perre, T. Duerinck, M. Maes, D. De Vos, E. Gobechiya, C. E. A. Kirschhock, G. V. Baron and J. F. M. Denayer, ChemSusChem, 2011, 4, 1074.

25 D. Fairen-Jimenez, S. A. Moggach, M. T. Wharmby, P. A. Wright, S. Parsons and T. Düren, J. Am. Chem. Soc., 2011, 133, 8900.

26 P.-S. Lee, J. Lee, N. Shin, K.-H. Lee, D. Lee, S. Jeon, D. Choi, W. Hwang and H. Park, Adv. Mater., 2008, 20, 1732. 